\title{
Impact of smoking status on workplace absenteeism and productivity
}

\author{
Michael T Halpern, Richard Shikiar, Anne M Rentz, Zeba M Khan
}

\begin{abstract}
Objectives-To: evaluate the impact of smoking status on objective productivity and absenteeism measures; evaluate the impact of smoking status on subjective measures of productivity; and assess the correlation between subjective and objective productivity measures.
\end{abstract}

Design-Prospective cohort study in a workplace environment.

Subjects-Approximately 300 employees (100 each of former, current, and never smokers) at a reservation office of a large US airline.

Main outcome measures-Objective productivity and absenteeism data were supplied by the employer. Subjective assessments of productivity were collected using a self report instrument, the Health and Work Questionnaire (HWQ).

Results-Current smokers had significantly greater absenteeism than did never smokers, with former smokers having intermediate values; among former smokers, absenteeism showed a significant decline with years following cessation. Former smokers showed an increase in seven of 10 objective productivity measures as compared to current smokers, with a mean increase of $4.5 \%$. While objective productivity measures for former smokers decreased compared to measures for current smokers during the first year following cessation, values for former smokers were greater than those for current smokers by 1-4 years following cessation. Subjective assessments of "productivity evaluation by others" and "personal life satisfaction" showed significant trends with highest values for never smokers, lowest for current smokers, and intermediate for former smokers.

Conclusions-Workplace productivity is increased and absenteeism is decreased among former smokers as compared to current smokers. Productivity among former smokers increases over time toward values seen among never smokers. Subjective measures of productivity provide indications of novel ways of productivity assessment that are sensitive to smoking status.

(Tobacco Control 2001;10:233-238)

Keywords: smoking cessation; workplace; absenteeism; productivity; questionnaires

Cigarette smoking has been identified as the single most preventable cause of both morbidity and premature death in the USA. In 1990, smoking attributable mortality in the USA was estimated as $418690 .{ }^{1}$ In addition to the morbidity and mortality resulting from smoking, the economic burden associated with smoking is substantial. A 1994 report estimated that the annual direct medical care cost attributable to smoking in 1993 was $\$ 50$ billion. $^{2}$ The costs attributable to smoking are particularly important to employers. Beyond increased medical care costs attributable to smoking, ${ }^{3}$ employers incur additional indirect costs for smoking employees. These include impacts on workplace absenteeism and productivity. ${ }^{4}$ The US Office of Technology Assessment estimated that in 1990 the workplace cost from disability and premature mortality caused by smoking was $\$ 47$ billion. ${ }^{5}$ Several studies have indicated that smoking employees have substantially greater absenteeism, injuries, and accidents than do non-smoking employees. ${ }^{6-10}$

However, absenteeism represents only part of the total indirect burden of smoking to employers. It is also possible that in addition to lost time as a result of illness, smokers are also less productive on the job. To capture the full effect and benefit of smoking cessation, productivity loss caused by smoking must be quantified. However, productivity assessment in general is often quite difficult; objective measures of workplace productivity are usually not present, and subjective values are primarily used. This problem is even more difficult in attempting to evaluate workplace productivity among smokers. Among individuals with acute or chronic conditions (including smoking related conditions), productivity may decrease because of the employee working while suffering from illness symptoms. However, smokers may have additional productivity decrements from taking more breaks to adhere to the smoking ritual. Further, smokers and workers with other types of addictions may deny that their addictions have any negative influence on productivity.

To evaluate objectively the impact of smoking status on both absenteeism and productivity, we have conducted a prospective study in a work environment where quantitative measures of these workplace behaviours are available. In addition to objective productivity measures, we also evaluated subjective assessment of productivity using a new questionnaire, the Health and Work Questionnaire.

\section{Methods}

This study was designed to quantify differences in productivity and absenteeism levels between current, former, and never smokers in the workplace. The primary objectives of this study are to evaluate employer reported productivity and absenteeism among current, former, and never 
smokers in the workplace. The study was planned as a prospective analysis of 300 employees at a reservation office of a major US airline. This study was initially planned as a pilot/feasibility study for a more comprehensive workplace based smoking cessation intervention; as such, sample sizes were not established to achieve statistical power. Objective productivity data were collected from the study site for the four month study period to assess prospectively subjective and objective results by smoking status. Subjective productivity data were collected from the study site at baseline and then again on a monthly basis over the next three months. In addition, to allow evaluation of absenteeism and productivity results over a longer period of time (and thus better assess the impact of time since smoking cessation), we also analysed objective workplace data for one year before the start of the study.

SUBJECTS

The targeted sample size was 300 subjects employed by the study site. (The study site contained 647 employees who would have been eligible to participate in the study.) The on-site study coordinator was instructed to attempt to enroll the first approximately 100 volunteers in each of the three smoking status groups: current, former, and never smokers. Study participants were enrolled on a first volunteered, first enrolled basis. No information was available on employees who did not participate in the study. Smoking categories are defined as follows:

- Smoker: subject who smokes an average of 15 or more cigarettes per day for the last year and has not quit for greater than three months during the previous year.

- Former smoker: subject who has quit smoking.

- Never smoker: a subject who has never smoked or who has not smoked $>100$ cigarettes in their lifetime.

These are the standard smoking category definitions used by the sponsoring organisation (GlaxoWellcome) in its clinical trials of smoking cessation adjunctive therapy. All smoking status information was based on self report, with no validation performed. Subjects volunteered to participate in the study, and were reimbursed $\$ 50$ for their efforts. They completed informed consent forms and agreed to release the attendance and productivity information collected by their employer. All collected information remained confidential and was shared with the employer in aggregate form only. No individual level information related to smoking status and productivity was provided to study participants or the employer. As all of the performance measures were routinely collected among both study participants and non-participants in the absence of this study, it is unlikely that the collection of these measures introduced any bias.

ASSESSMENT OF ABSENTEEISM

The reservation centre collected objective measures on number of attendance interruptions, absenteeism days, and lost hours caused by both absenteeism and work injury. Attendance interruptions refer to periods of continuous absence. For example, an employee missing work on Monday and Tuesday has two days of absenteeism but one attendance interruptions, while an employee missing work on Monday and Wednesday has two days of absenteeism and two attendance interruptions. Data were collected for the four months that subjects were enrolled in the study. We also received the data on the same measures for the previous year. For this study, only the absenteeism data on attendance interruptions and lost days for the study period and one year previous were used in the analyses. Also, only the number of days lost because of work injury during the study period was used in the analyses. Information collected during the study period allowed us to evaluate both objective and subjective data collected in a uniform fashion, while data from the year before study initiation permitted greater evaluation of the impact of time since smoking cessation.

OBJECTIVE ASSESSMENT OF PRODUCTIVITY Beyond absenteeism information, the reservation centre collects other objective productivity measures based on an employee's performance. Measures involve the following productivity markers.

(1) Revenue calls handled-The number of calls leading to a sale handled per month by an employee.

(2) Ticket delivery system segments-The number of flight segments arranged by reservation personnel for delivery.

(3) Call work time-The average time a reservation clerk is unavailable between calls.

(4) Hours lost-The average time a reservation clerk is unavailable between calls without a sanctioned excuse.

(5) Potential flown segments - The total number of flight segments booked in the past scheduled to fly during the time period reviewed.

In addition, five measures of productivity are assessed as performance ratios. These are:

(1) Dollar amount per revenue call handled.

(2) Dollar amount per productive sign-in time.

(3) Potential flown segments per revenue call handled.

(4) Potential flown segments per productive sign-in time.

(5) Ticket delivery system segments per productive sign-in time.

These measures are assessed both on an individual level and relative to the department shift average (DSA) on a quarterly basis. For the analysis, we used only values that had been standardised relative to the DSA. This permitted comparisons among study participants on different working schedules, controlling for any baseline differences caused by the different shifts.

The summary productivity measure is a derived performance point total. This measure is used to determine changes in annual compensation for reservation employees; differences in a single performance point may determine whether an employee receives a raise and the magnitude of the raise. Points are 
evaluated quarterly among all employees and averaged annually. One hundred points are determined in each evaluation, 70 based on objective criteria and the remaining 30 based on subjective evaluations. Higher performance point values indicated higher levels of performance. For this analysis, only the 70 points based on objective criteria were examined.

SUBJECTIVE ASSESSMENT OF PRODUCTIVITY

The Health and Work Questionnaire (HWQ) was developed to assess productivity subjectively. A direct, subjective estimate of productivity loss caused by smoking is not advisable, both because there is a strong social desirability component motivating smokers to report otherwise, as well as the lack of an appropriate reference point against which long term smokers could compare their changes in productivity. The HWQ was developed to assess various aspects of productivity without completely relying on direct subjective estimation. Unlike other productivity assessment tools, the HWQ was designed to be a multidimensional measure of productivity. A brief summary of the HWQ is presented below. While comparisons between objective productivity values and results from the HWQ have been performed, this instrument has not yet been validated. Copyright of the HWQ is owned by the GlaxoSmithKline Group of Companies (C2000). Copies of the HWQ and the factor analysis used to develop its subscales are available from one of the authors (see Acknowledgements).

The HWQ consists of 24 questions, several of which were multi-part questions, comprising six subscales. Six subscales were identified via factor analysis of the baseline data, accounting for $69 \%$ of the variance of the correlation matrix. The six subscales are: productivity; impatience and irritability; concentration and focus; work satisfaction; satisfaction with supervisor; and personal life satisfaction. To try to minimise the "social desirability" tendencies on the part of the respondent, we asked respondents to rate their work quality, quantity, and efficiency from their supervisor's and their co-worker's perspective, as well as from their own perspective.

All items have a 10 point response scale, tailored to each question (for example, "very dissatisfied" to "very satisfied" for questions dealing with work satisfaction; "my worst ever" to "my best ever" for questions dealing with rating quantity, quality, and efficiency of work).

Table 1 Study population demographic characteristics *

\begin{tabular}{llll}
\hline & $\begin{array}{l}\text { Current smokers } \\
(n=95)\end{array}$ & $\begin{array}{l}\text { Former smokers } \\
(n=86)\end{array}$ & $\begin{array}{l}\text { Never smokers } \\
(n=111)\end{array}$ \\
\hline $\begin{array}{l}\text { Mean (SD) age } \\
\text { Sex (\%) }\end{array} \quad 38(10.4)$ & $43.7(8.9)$ & $36.8(9.4)$ \\
$\quad$ Male & 22 & 13 & 12 \\
$\quad$ Female & 78 & 87 & 88 \\
Race/ethnicity (\%) & 79 & 84 & 69 \\
$\quad$ White & 15 & 9 & 24 \\
$\quad$ Black & 0 & 1 & 0 \\
$\quad$ Asian & 4 & 3 & 3 \\
$\quad$ American Hispanic & 2 & 2 & 4 \\
$\quad$ Other & 83 & 76 & 78 \\
Full time employment (\%) & & & \\
\hline
\end{tabular}

^No significant differences by smoking status as determined by ANOVA.
Subscale scores are derived by averaging items within a subscale. The internal consistency reliability (Cronbach's $\alpha$ ) for the subscale ranged from 0.72 for "impatience and irritability" to 0.96 for "productivity". Except for the "impatience and irritability" subscale, the reliability of each subscale was greater than 0.8. The reliability for the summary HWQ score was 0.81 .

In the study reported here, the HWQ was preceded by two other series of questions. The first series (17 questions) ascertained smoking history as well as age, sex, and ethnicity. The second series consisted of 13 questions, inquiring about incidence of cold or cold-like symptoms during the past week, days absent from work because of these symptoms, overall health, prescription medications taken during the week, and mental health issues such as feelings of anxiety, depression, or panic. This second series of questions were not the subject of analyses in the present manuscript.

\section{DATA ANALYSES}

All data were converted to an SAS file for analysis. Routine edit checks were completed to identify and resolve data inconsistencies. Standard frequency distributions and crosstabulations of variables were generated to ensure data consistency.

Data were analysed using PC SAS (SAS Institute, Cary, North Carolina, USA). Descriptive analyses included means, standard deviations, and ranges, or frequency distributions. Cross-tabulations were performed on variables by smoker category (current, former, never). Comparisons across study participants of three smoking status groups (never, former, and current smokers) were made using analysis of variance (ANOVA), while comparisons between two groups (former versus current smokers) were performed using $t$ tests. The impact of time since smoking cessation on absenteeism was determined using multivariate ordinary least square regression. Study participants with a missing value for a particular absenteeism or productivity measure were excluded from the analysis of that measure; no missing values were imputed.

\section{Results}

Table 1 presents the demographic characteristics of the study population. The current smoker group had a greater proportion of males and full time (versus part time) employees than the other two groups. Former smokers tended to be slightly older than the other groups, while never smokers included a greater proportion of minority individuals. However, the differences among the three smoking status groups were not significant for any of these variables.

Table 2 presents absenteeism results by smoking status. This table presents the mean, standard deviation, and range of absenteeism values for each smoking status group. Statistics were performed to evaluate differences across the three groups. Current smokers had greater absenteeism than did never smokers during the study period, as measured by either absenteeism 
Table 2 Absenteeism by smoking status

\begin{tabular}{|c|c|c|c|c|}
\hline Variable & Current smokers & Former smokers & Never smokers & $p$ Value * \\
\hline Total study absenteeism days caused by sickness $\dagger$ & $3.99(4.86)$ & $2.40(3.54)$ & $1.33(2.20)$ & 0.0001 \\
\hline Range & $0-24$ & $0-14$ & $0-9$ & \\
\hline Total number of attendance interruptions caused by sickness $\dagger$ & $0.89(0.89)$ & $0.59(0.79)$ & $0.43(0.66)$ & 0.0002 \\
\hline Range & $0-3$ & $0-3$ & $0-3$ & \\
\hline Absenteeism days caused by sickness during previous year $\dagger$ & $6.16(7.51)$ & $4.53(7.20)$ & $3.86(5.32)$ & 0.043 \\
\hline Range & $0-54$ & $0-38$ & $0-24$ & \\
\hline Number of attendance interruptions caused by sickness during previous year $\dagger$ & $1.72(1.58)$ & $1.09(1.40)$ & $1.14(1.43)$ & 0.0053 \\
\hline Range & $0-9$ & $0-6$ & $0-6$ & \\
\hline Total study absenteeism days caused by work injury $\dagger$ & $0.32(2.70)$ & $0.06(0.54)$ & $0.21(2.00)$ & NS \\
\hline Range & $0-26$ & $0-5$ & $0-21$ & \\
\hline
\end{tabular}

^As determined by ANOVA.

†Mean (SD) presented.

days or attendance interruptions. Former smokers had intermediate absenteeism values except for number of attendance interruptions caused by sickness during the previous year and total study days lost because of work injury, where former smokers had the lowest values. Results were similar for the year before the study period: current smokers had the greatest rate of absenteeism, never smokers had the lowest rate, and former smokers were intermediate. Differences in absenteeism were significant. As presented in the last row of table 2, former smokers had the lowest rate of study days lost because of work injuries. However, all work injury related absenteeism values were small, and these differences were not significant.

Table 3 presents regression analysis results evaluating the impact of time since smoking cessation on absenteeism rates. Multivariate ordinary least squares regression was performed on current and former smokers, controlling for age, sex, full time/part time employment status, and time since smoking cessation (set to zero for current smokers). Using data from the study period, former smokers experienced a decrease in 0.1 absenteeism days and 0.017 attendance interruptions for every year of smoking cessation. Similar results were observed for regression analysis of data from the year before the study: former smokers experienced significant decreases of 0.15 absenteeism days and 0.045 attendance interruptions for each year since cessation. The impact of years of cessation was significant for all of these absenteeism measures with $\mathrm{p}<0.05$.

Objective productivity measures by smoking status were also examined. As presented in the Methods section, data were provided on 10 productivity categories: five absolute measures for each study participant and five relative measures, comparing each study participant's productivity to that of the relevant co-workers (the DSA). Compared to current smokers, former smokers were more productive for 7 of the 10 categories. The difference in productivity for former smokers (as compared to current smokers) ranged from improvement of $19 \%$ to worsening of approximately $6 \%$. The average across all five absolute measures indicated a higher productivity level of over $7 \%$ for former smokers compared to current smokers. The percentage of difference in productivity in the relative measures was smaller than that observed in the absolute measures, but the average across these five measures indicated improved productivity of former smokers by $1.72 \%$. Across all 10 objective measures, the mean percentage increase for former smokers compared to current smokers was approximately $4.5 \%$. None of the productivity differences between current and former smokers or current and never smokers reached significance.

As discussed in the Methods section, the summary productivity measure is a derived performance point total. We evaluated total performance points by smoking status. The mean performance points for never smokers (36.0) was greater than that for current smokers (34.6), with higher values reflecting greater objective productivity. Former smokers had a mean total performance points of 37.5, greater than that for either never or current smokers. We also evaluated the impact of time since smoking cessation on former smokers' performance score. Among former smokers who have quit smoking for less than one year, the mean total performance points (24.1) is lower than that for current smokers. However, by one to five years following cessation, the former smoker mean performance value (40.7) is higher than those of current smokers, reflecting greater objective productivity. This value remains fairly consistent; following five or more years of cessation, the mean total performance points for former smokers is 39.8 . Differences in total performance points between current and former smokers or current and never smokers did not reach significance.

Table 3 Absenteeism and years of smoking cessation

\begin{tabular}{llll}
\hline Measure & Current smokers & Former smokers & Change per year of cessationt \\
\hline Study absenteeism days caused by sickness & 3.99 & 2.4 & -0.1 \\
Study attendance interruptions caused by sickness & 0.89 & 0.59 & -0.017 \\
One year absenteeism days caused by sickness & 6.16 & 4.53 & -0.15 \\
One year attendance interruptions caused by sickness & 1.72 & 0.03 & -0.045 \\
\hline
\end{tabular}

^Mean value.

†Analysis performed using multivariate ordinary linear least squares regression, controlling for age, sex, full time/part time employment status, and time since smoking cessation. Only current and former smokers were included in the analysis, as time since cessation would be undefined for never smokers. A zero value for time since cessation was assigned to current smokers.

$\neq \mathrm{p}$ Value refers to the significance of the independent variable "years of cessation" in the regression model. 
Table 4 Relations between HWQ scores and smoking status

\begin{tabular}{|c|c|c|c|c|c|c|c|c|c|c|}
\hline \multirow[b]{2}{*}{ Subscale } & \multicolumn{3}{|c|}{ Current smokers } & \multicolumn{3}{|c|}{ Former smokers } & \multicolumn{3}{|c|}{ Never smokers } & \multirow[b]{2}{*}{$p$ Value $^{\star}$} \\
\hline & $n$ & Mean & $S D$ & $n$ & Mean & $S D$ & $n$ & Mean & $S D$ & \\
\hline Productivity & 96 & 7.605 & 1.758 & 87 & 7.695 & 1.288 & 111 & 8.054 & 1.291 & 0.065 \\
\hline Assessment of own performance & 96 & 7.483 & 1.751 & 87 & 7.375 & 1.373 & 111 & 7.771 & 1.377 & 0.156 \\
\hline Assessment by others & 96 & 7.707 & 1.899 & 87 & 7.962 & 1.324 & 111 & 8.288 & 1.343 & 0.026 \\
\hline Concentration/focus & 95 & 7.148 & 2.201 & 87 & 6.968 & 2.314 & 111 & 7.306 & 1.992 & 0.551 \\
\hline Supervisor relations & 96 & 7.818 & 2.090 & 87 & 7.753 & 1.888 & 111 & 8.162 & 1.977 & 0.287 \\
\hline Personal life satisfaction & 96 & 7.750 & 2.103 & 87 & 8.092 & 1.395 & 111 & 8.431 & 1.442 & 0.015 \\
\hline Work life satisfaction & 96 & 7.034 & 2.049 & 87 & 6.724 & 1.686 & 111 & 7.205 & 1.549 & 0.163 \\
\hline Impatience/irritability & 96 & 8.174 & 1.558 & 87 & 8.215 & 1.419 & 111 & 8.369 & 1.395 & 0.593 \\
\hline \multicolumn{11}{|l|}{ Total score } \\
\hline Across items & 96 & 7.563 & 1.528 & 87 & 7.534 & 1.373 & 111 & 7.857 & 1.255 & 0.182 \\
\hline Across subscales & 96 & 7.588 & 1.527 & 87 & 7.616 & 1.275 & 111 & 7.921 & 1.247 & 0.115 \\
\hline
\end{tabular}

${ }^{\star}$ p Values were derived from ANOVA comparing the three smoking status groups. Pairwise comparisons of HWQ scores for former and current smokers (not presented) did not yield any significant differences.

Table 4 present results from subjective productivity assessment using the HWQ. It presents results from the ANOVA of HWQ subscales by smoking status. Probability values provided in this table represent statistical differences comparing subscale scores across all three smoking status groups; there are two significant differences. First, assessment of productivity by others was significant $(p=0.026)$, with highest assessment scores among never smokers, lowest among current smokers, and intermediate scores for former smokers. A similar pattern was also seen in the personal life satisfaction subscale $(p=0.015)$. Differences in the overall productivity subscale scores, which include productivity assessment both by self and by others, approached significance $(p=0.065)$. Pairwise comparisons of current and former smokers did not yield any significant differences.

As significant differences were observed in the productivity assessment-by-others subscale, we examined differences between current and former smokers for individual items comprising this subscale. Figure 1 presents results from the six items in the productivity assessment by others subscale. These six items capture self reported productivity assessment in three dimensions (efficiency, quality, and amount of work) from two external perspectives (supervisors and co-workers). While none of the pairwise comparisons produced significant differences,

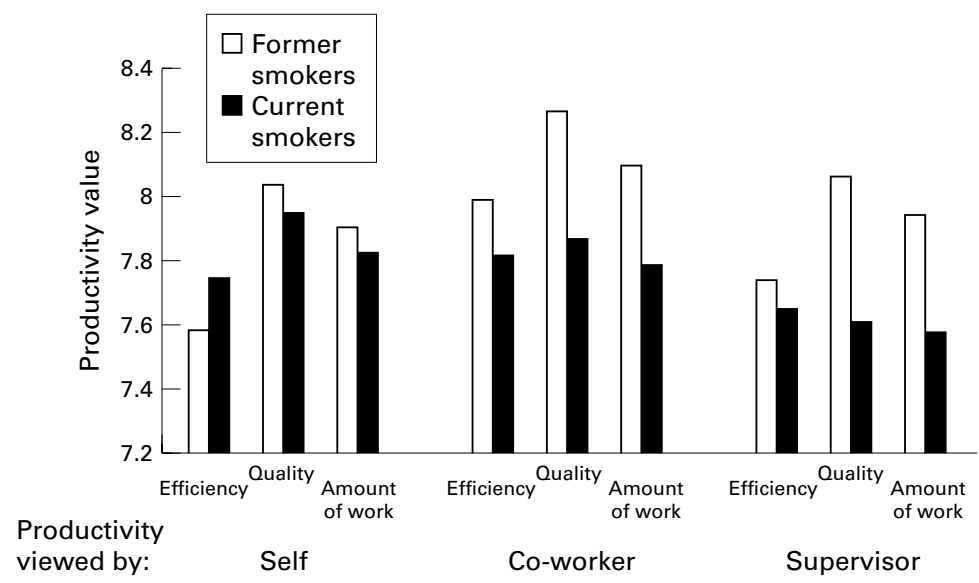

Figure 1 HWQ items in assessment by self and assessment by others productivity subscales by smoking status. None of the pairwise comparisons produced significant differences. Differences in the items dealing with quality reported by supervisors or by co-workers approached significance ( $p=0.10$ and 0.09 , respectively). former smokers indicated higher productivity levels (as assessed by others) as compared to current smokers for all six items. Differences in the items dealing with quality reported by supervisors or by co-workers approached significance ( $\mathrm{p}=0.10$ and 0.09 , respectively).

\section{Discussion}

This analysis has evaluated differences in absenteeism, objectively assessed productivity, and subjectively assessed productivity by smoking status in a defined workplace cohort. The small size of the study population limits our ability to assess significant differences. However, changes in absenteeism did differ significantly in comparing never, former, and current smokers. The impact of time since smoking cessation on absenteeism was also significant when comparing only former and current smokers, suggesting a "dose-response" relationship between years of cessation and absenteeism. Objective productivity measures did not significantly differ across the smoking status groups, but trends were observed indicating greater productivity among former versus current smokers and increased productivity with time since smoking cessation. Subjective productivity measured showed a small number of significant differences or trends with smoking status. It is of particular interest that differences by smoking status were not observed when respondents were asked to rate their own productivity; rather, significant differences in productivity subscales were seen only when respondents were asked to specify how others (co-workers or supervisors) would rate their productivity.

There are a number of limitations for this study, mainly related to the study population. In order to capture complete absenteeism and productivity information, we chose a cohort of reservation agents working for a major US airline. The reservation agents are younger than the mean US workforce age, and a larger proportion is female. These workers are highly monitored during the course of the duties, and are aware of the impact of their productivity on remuneration. As such, their productivity values may not correspond to that of workers in other fields or work environments, similar to the Hawthorne effect observed for health behaviours of individuals in health outcomes studies. Further, the tasks for reservation agents involve a defined and specific set of activities. Because of these factors, generalisation of the findings in this 
study to other types of workers or work environments must be performed with care. However, the results present internally consistent information on the effect of smoking status on absenteeism and productivity. Also, as noted above in Methods, this study was initially planned as a pilot/feasibility study for a more comprehensive workplace based smoking cessation intervention; as such, sample sizes were not established to achieve statistical power.

Previous assessments of productivity losses among smokers have primarily been based upon assumptions. In a model of worksite smoking cessation programmes, Warner and colleagues ${ }^{11}$ assumed a decrease in productivity of $1 \%$ (five minutes per eight hour day for smokers) as a base case. Kristein ${ }^{8}$ made a similar assumption as a "reasonable underestimate" for productivity losses by smokers, while Weis $^{12}$ assumed 35 minutes per day due to smoking breaks. A number of studies have provided indirect assessment of the productivity impact of smoking. Robin and Weir ${ }^{13}$ reported that smoking by dentists was associated with decreased motor performance and dexterity and increased fatigue, while Barad $^{14}$ reported that over $50 \%$ of non-smokers experienced difficulty working near a smoker. The present study is the first published attempt to quantify the productivity impact of smoking using both subjective and objective data.

Other studies have shown that the cost of absenteeism and decreased productivity caused by medical conditions can be substantial. For example, Greenberg and colleagues $^{15}$ estimated the societal costs of absenteeism and decreased productivity for depressed employees were each approximately $\$ 12$ billion (in 1990). Similarly, Stoller ${ }^{16}$ estimated that the 1990 societal productivity loss caused by insomnia exceeded $\$ 40$ billion. The results of our study show that former smokers and never smokers may indeed be more productive than current smokers; this may result in even greater societal costs being attributable to smoking.

In future research, it will be important to assess the generalisability of these results by assessing productivity by smoking status among diverse types of workers. Objective measures constitute a "gold standard" for workplace productivity assessment. However, as many (or most) occupations do not lend themselves to objective evaluation of productivity, use of a subjective instrument that has undergone at least preliminary evaluation will provide a basis for quantitative assessments. We do not believe that the HWQ can completely substitute for objective productivity measures, and this instrument has not yet been validated. However, the differences seen by smoking status among this small study population suggest that the HWQ may be useful for projecting certain aspects of productivity, especially when objective measures cannot be assessed. With growing concerns regarding the cost of medical care and the "value for money" of new preventive and therapeutic interventions, the ability to access the full cost of health care conditions, including their

\section{What this paper adds}

Beyond increased medical care costs attributable to smoking, employers incur indirect costs (impacts on workplace absenteeism and productivity) for smoking employees. Evaluating the impact of smoking status on workplace productivity is particularly difficult; objective productivity measures are usually not present and subjective values or estimates are primarily used.

We conducted a prospective study in a work environment where quantitative measures of absenteeism and productivity were available. Current smokers had significantly greater absenteeism than did never smokers; former smokers had intermediate values and showed a significant decline in absenteeism with years following cessation. Former smokers showed increases in a majority of objective productivity measures compared to current smokers; former smokers' total productivity was greater than current smokers' by 1-4 years following cessation. These findings indicate that former smokers show decreased indirect costs compared to current smokers over a relative brief period, and that smoking cessation may well decrease employer costs.

impact on workplace productivity, will become increasingly important.

The authors wish to thank GlaxoWellcome for funding the research reported in this paper. Requests for permission to use the Health and Work Questionnaire (HWQ) should be directed to Dr Zeba Khan, GlaxoSmithKline, Five Moore Dr., Research Triangle Park, NC 27709 (zmk85058@gsk.com).

1 US Department of Health and Human Services. Cigarette smoking-attributable mortality and years of potential life lost-United States 1990. MMWR Morb Mortal Wkly Rep 1993;42:645-9

2 US Department of Health and Human Services. Medicalcare expenditures attributable to cigarette smoking-
United States, 1993. MMWR Morb Mortal Wkly Rep 1994;43:469-72.

3 Penner M, Penner S. Excess insured health care costs from tobacco-using employees in a large group plan. $\mathcal{f}$ Occup Med 1990;32:521-3.

4 MacKenzie TD, Bartecchi CE, Schrier RW. The human costs of tobacco use (second of two parts). N Engl f Med 1994;380:975-80.

5 Office of Technology Assessment. Smoking-related deaths and financial costs: estimates for 1990, revised ed. Washington DC: Office of Technology Assessment, 1993.

6 Batenburg M, Reinken JA. The relationship between sickness absence from work and pattern of cigarette smoking. NZ Med f 1990;103:10-13.

7 Hocking B, Grain H, Gordon I. Cost to industry of illnesses related to alcohol and smoking. A study of Telecom Australia employees. Med f A ust 1994; 161:407-11.

8 Kristein MM. How much can business expect to profit from smoking cessation? Prev Med 1983;12:358-81.

9 Ryan J, Zwerling C, Orav EJ. Occupational risks associated with cigarette smoking: a prospective study. Am F Public Health 1992;82:29-32.

10 Ryan J, Zwerling C, Jones $M$. Cigarette smoking at hire as a predictor of employment outcome. F Occup Environ Med 1996;38:928-33.

11 Warner KE, Smith RJ, Smith DG, et al. Health and economic implications of a work-site smoking cessation program: A simulation analysis. f Occup Environ Med program: A simulat

12 Weis WL. Can you afford to hire smokers? Personnel Administration 1981;26:71-8.

13 Robin GN, Weir JM. The cost of smoking to a dental practice and to dentistry. F Am Dent Assoc 1970;80:598-600. 14 Barad CB. Smoking on the job: the controversy heats up. Occup Health Safety 1979;48:21-4.

15 Greenberg PE, Stiglin LE, Finkelstein SN, et al. The economic burden of depression in 1990. $\mathcal{F}$ Clin Psychiatry 1993;54:405-18.

16 Stoller, KS. Economic effects of insomnia. Clin Ther 1994;16:873-97. 